\title{
Novel perspectives in clinical cardiology and cardiac surgery
}

\author{
Anastasia S Mihailidou*

\begin{abstract}
4th International Conference on Clinical \& Experimental Cardiology, San Antonio, TX, USA, 14-16 April 2014
\end{abstract}

\begin{abstract}
The International Conference on Clinical \& Experimental Cardiology was held in San Antonio (TX, USA) on 14-16 April 2014. This was the fourth meeting and had the theme 'Novel Perspectives on Clinical Cardiology and Cardiac Surgery' with sessions in heart disease, congenital heart disease, cardiac therapeutic agents, biophysics and systems biology, current research, and interventional cardiology, providing an interactive forum for discussion of science and clinical practices. Presentations by delegates from Africa, Saudi Arabia, India, China, Japan, Australia, Europe, South America, in addition to Canada and the USA, provided an opportunity for collaboration but also an appreciation of the challenges for treatment in remote locations as well as distance between health facilities.
\end{abstract}

Currently there are approximately 15 million people with ischemic heart disease (IHD) in the USA [1], while prevalence of IHD in India has increased from 2\% in year 1960 to $10.5 \%$ in 2000, which equates to 30 million people and increasing [2]. Since IHD can progress to heart failure and the increasing aging population globally, a major health burden is emerging and novel perspectives in treatment strategies are required. A key focus for many of the presentations was inflammation, which highlights the increasing recognition of the important role it plays in cardiovascular disease. The inflammatory networks are complex and can be divided into an acute inflammatory response to infectious agent or tissue injury or chronic inflammation, which is reported in many conditions, including aging, diabetes and atherosclerosis.

Proinflammatory markers in atherosclerosis include cytokines, monocytes, smooth muscle cells, perivascular adipose tissue and low density lipoprotein (LDL)-cholesterol. There is increasing interest in another trigger, cholesterol-rich bacteria such as Chlamydophila pneumoniae, Mycoplasma pneumonia and Archeae, which have recently been identified inside the unstable plaque [3]. These bacteria trigger inflammation by facilitating release of cytokines leading to degradation of collagen tissue and promoting rupture of the plaque, a finding that has been confirmed by others [4], who have found 16-times higher bacterial DNA from Chlamydia pneumonia and other dental bacteria in aspirated thrombi than from blood samples of patients with myocardial infarction. Hence, a systems approach to treatment for cardiovascular disease is encouraged.

Oxidative stress plays a key role in many inflammatory conditions, including atherosclerosis. Recent studies show that metabolic stress increased expression levels of NADPH oxidase 4 (Nox4) in monocytes to enhance macrophage recruitment and progress atherosclerosis [5], although the exact proinflammatory mechanism(s) were not defined. To define the cellular targets required, reverse translation to bench studies in monocytes. Prolonged exposure to LDL, high glucose $(\mathrm{HG})$ concentrations or a combination of both activated Nox4 expression while increasing

${ }^{*}$ Cardiovascular \& Hormonal Research Laboratory, Department of Cardiology, Kolling Institute, Royal North Shore Hospital \& University of Sydney, Sydney, NSW 2065, Australia; Tel.: +61 2992 64956; Fax: + 612922 64044; anastasia.mihailidou@sydney.edu.au

\section{KEYWORDS}

- atherosclerosis $\bullet$ atrial fibrillation • inflammation - ischemic heart disease - serpins 
intracellular $\mathrm{H}_{2} \mathrm{O}_{2}$ formation, promoting $S$-glutathionylation of MKP-1 and actin, resulting in accelerated monocyte chemotaxis via ERK and p38MAPK signalling and increased actin remodelling. This novel proinflammatory mechanism may be a potential treatment target, especially in metabolic disorders such as diabetes and obesity.

Modification of activity of serine protease inhibitors (serpins) was presented as an alternative approach for targeting inflammatory pathways. Serpins are an extensively distributed family of protease inhibitors that regulate coagulation and thrombolytic pathways as well as inflammation. Serpins in which their function has been identified include SERPINC1, which is antithrombin, SERPINE1 or better recognized as plasminogen activator inhibitor 1 (PAI 1), as well as SERPINA6 or corticosteroid-binding globulin, SERPINA7 (thyroxine-binding globulin) and SERPINA8 (angiotensinogen), among many others [6]. In experimental studies, viral and mammalian serpin reactive center loop (RCL) peptides reduced plaque growth and show potential for reducing inflammation [7]. The RCP loop is the region that undergoes conformational change to interact with the target proteases and therefore these proteins provide a new approach to anti-inflammatory therapy.

Atrial fibrillation (AF) is increasing globally [8] with the increasing aging population, with an estimated 1 in 4 people likely to develop $\mathrm{AF}$ in their lifetime and a significant health burden given that the presence of AF increases the risk of thromboembolic stroke by fivefold. Inflammation and thrombogenesis have a significant role at the onset of AF and as an indicator of future episodes [9,10], with increased markers of platelet activation, coagulation, inflammation and endothelial dysfunction measured. Interestingly these markers have been found to differ when measured from the left and right sides of the heart, consistent with patterns of thrombus formation in the left but not right atrium [11], although the mechanisms have not been fully defined. A recent study identified that there is differential cardiac-specific platelet activation and reactivity $[9,12]$. Since platelets also release inflammatory mediators and cytokines, activation of inflammation may further promote thrombus formation. Therefore, the mechanisms for the role of inflammatory pathways in many cardiac conditions is increasingly being investigated, as well as specific treatment strategies to provide the potential for improved clinical management.

Financial \& competing interests disclosure ASM was Moderator for this meeting and is a member of the Organising Committee. The author has no other relevant affliations or financial involvement with any organization or entity with a financial interest in or financial conflict with the subject matter or materials discussed in the manuscript apart from those disclosed.

No writing assistance was utilized in the production of this manuscript.

\section{References}

1 Go AS, Mozaffarian D, Roger VL et al. Heart disease and stroke statistics - 2013 update: a report from the American Heart Association. Circulation 127(1), e6-e245 (2013).

2 H. N. Kumar H. A retrospective study on the profile of ischemic heart disease [IHD] cases admitted in KMC Attavar, Mangalore. J. Clin. Exp. Cardiolog. 5, 3 (2014).

3 Ramires, JAF. Atherosclerosis: Infection \& Inflammation involvement, the continuous discussion. J. Clin. Exp. Cardiolog. 5(3), 41 (2014).

4 Pessi T, Karhunen V, Karjalainen PP et al. Bacterial signatures in thrombus aspirates of patients with myocardial infarction. Circulation 127(11), 1219-1228 (2013).
5 Lee CF, Ullevig S, Kim HS, Asmis R. Regulation of monocyte adhesion and migration by Nox4. PLoS ONE 8(6), e66964 (2013).

6 Law RHP, Zhang Q, McGowan S, Buckle AM. An overview of the serpin superfamily. Genome Biol. 7(5), 216 (2006).

7 Chen H, Davids JA, Zheng D et al. The serpin solution; targeting thrombotic and thrombolytic serine proteases in inflammation. Cardiovasc. Hematol. Disord. Drug Targets 13(2), 99-110 (2013).

8 Chugh SS, Havmoeller R, Narayanan K. Worldwide epidemiology of atrial fibrillation. Circulation 129, 837-847 (2014).

9 Lim HS, Willoughby SR, Schultz C et al. Effect of atrial fibrillation on atrial thrombogenesis in humans: impact of rate and rhythm. J. Am. Coll. Cardiol. 61(8), 852-860 (2013).

10 Issac TT, Dokainish H, Lakkis NM. Role of inflammation in initiation and perpetuation of atrial fibrillation: a systematic review of the published data. J. Am. Coll. Cardiol. 50(21), 2021-2028 (2007).

11 Schultz CD, Rangneker G, Lim HS et al. Characterization of thrombogenic, endothelial and inflammatory markers in supraventricular tachycardia: a study in patients with structurally normal hearts. Clin. Exp. Pharmacol Physiol. 41(8), 551-557 (2014).

12 Willoughby SR, Roberts-Thomson RL, Lim HS et al. Atrial platelet reactivity in patients with atrial fibrillation. Heart Rhythm 7(9), 1178-1183 (2010). 